\title{
ChatBots and Virtual Assistants
}

\author{
S V Sai Abitha ${ }^{1}$ \\ ${ }^{1}$ JSS Academy of Technical Education, Bangalore
}

May 10, 2021

S V Sai Abitha 13/06/2020

Computer Science and Engineering

ChatBots and Virtual Assistants

Abstract - ChatBot can be described as software that can chat with people using artificial intelligence. The use of chatbots evolved rapidly in numerous fields in recent years, including Marketing, Supporting Systems, Education, Health Care, Cultural Heritage, and Entertainment.

In this paper I first present the overview of chatbots. Next we discuss the need of chatbots and virtual assistants and its usefulness in a variety of areas. We also clarify the necessary technological concepts. Then we move on to classification of chatbots based on various criteria, such as the area of knowledge they refer to, the need they serve and others. Furthermore I present the general architecture of modern chatbots.

Keywords : Chatbot . Chatbot Architecture . Artificial Intelligence . NLP

\section{Introduction}

Artificial intelligence (AI) refers to the simulation of human intelligence in machines that are programmed to think like humans and mimic their actions. (AI) increasingly integrates our daily lives with the creation and analysis of intelligent software and hardware, called intelligent agents. Intelligent agents can do a variety of tasks ranging from labor work to sophisticated operations.

A chatbot is a typical example of an AI system. Chatbots can mimic human conversation and entertain users but they are not built only for this. They are useful in applications such as education, information retrieval, business, and e-commerce. They became so popular because there are many advantages of chatbots for users and developers too. Most implementations are platform-independent and instantly available to users without needed installations.

Moreover, payment services are integrated into the messaging system and can be used safely and reliably and a notification system re-engages inactive users. Chatbots are integrated with group conversations or shared just like any other contact, while multiple conversations can be carried forward in parallel. Knowledge in the use of one chatbot is easily transferred to the usage of other chatbots, and there are limited data requirements.

Communication reliability, fast and uncomplicated development iterations, lack of version fragmentation, and limited design efforts for the interface are some of the advantages for developers too.

The rest of the paper is organized as follows. In Sect. 2, the chatbot trend is described while in Sect. 3, essential concepts relevant to chatbot technology are described. Next, in Sect. 4, we present a classification of existing chatbots while in Sect. 5, we present the underlying chatbot architecture. Finally, Sect. 6 report's conclusion. 


\section{The Chatbot Trend}

In the very initial entry of chatbot, people were curious and anxious about the influence of this technology and how it's going to affect the future of enterprise and business. Chatbots are trending among the industrial sectors. Industries use chatbots to automate the business processes, hence targeting smart work than hard work. Chatbots are transforming the way businesses communicate with their customers. According to statistics in $201969 \%$ of consumers prefer chatbots because of their ability to provide fast answers to simple questions. Over $50 \%$ of customers expect a business to be open $24 / 7$ and $95 \%$ of consumers believe that 'customer service' will benefit the most from chatbots. $67 \%$ of customers globally reported using a chatbot for customer support in the past year. $37 \%$ of consumers expect to receive quick responses during emergencies. $33 \%$ of consumers would like to use chatbots to make reservations, place online orders, and other functions. The top industries profiting from chatbots are real estate, travel, education, healthcare. For any industry type, if you take account the user base, there are some basic questions that users generally ask. And for these specific question, same specific answers are required to be answered every time the customer makes the similar query. For such purposes, chatbot is the best engaging way to answer these common questions. Chatbots can be used to assist users or consumers for a specific task, whenever they want. And as chatbots do not get tired or bored, hence can be employed to provide customer service round the clock. One of the great advantages of chatbot is, it requires less development cost in comparison to application development. Well, making an investment into a quality product like chatbot is worth. Chatbots are going mainstream across diverse business processes and user applications. Chatbots are growing in all directions, then be it a messaging app, social platforms or other product and service-based websites.

\section{Essential Concepts}

Below are some fundamental concepts related to chatbot technology.

AI is wide-ranging branch of computer science concerned with building smart machines capable of performing tasks that typically require human intelligence. Artificial Intelligence gives a human touch to every conversation chatbot strikes. The bot understands the user's query and triggers an accurate response. The way humans are able to understand each other's concern and give a response accordingly.

The Artificial Intelligence Markup Language (AIML) was created from 1995 to 2000, and it is based on the concepts of Pattern Recognition or Pattern Matching technique. It is applied to natural language modeling for the dialogue between humans and chatbots that follow the stimulus-response approach. It is an XML-based markup language and it is tag-based.

Natural Language Processing (NLP), an area of artificial intelligence, explores the manipulation of natural language text or speech by computers. Knowledge of the understanding and use of human language is gathered to develop techniques that will make computers understand and manipulate natural expressions to perform desired tasks. Most NLP techniques are based on machine learning.

Pattern Matching is predicated on representative stimulus-response blocks. A sentence (stimuli) is entered, and output (response) is created consistent with the user input.

Natural language understanding (NLU) is a branch of artificial intelligence (AI) that uses computer software to understand input made in the form of sentences in text or speech format. NLU directly enables human-computer interaction (HCI). NLU understanding of natural human languages enables computers to understand commands without the formalized syntax of computer languages and for computers to communicate back to humans in their own languages.

\section{Types of Chatbots}

Chatbots can be classified using different parameters

Scripted/Quick Reply Bots - A scripted chatbot, as the name suggests, is one who's interaction with the end customer happens through a pre-defined knowledge base and technical capabilities that can aptly 
respond only to specific instructions. In that case, the queries must be aligned with the language programmed in the bot.

NLP Chatbots - Chatbots, in general, are counted as one of the biggest applications of Artificial Intelligence (AI). At its core, it utilizes an AI technology- Natural Language Processing (NLP) to map user input (text or voice) to an intent. NLP chatbots classify the messages and then language parsing is done to get variables for an answer.

Social Messaging Chatbots - These chatbots are integrated within a social messaging platform (Messenger, Telegram, Whatsapp, Slack etc.), making it easy for customers to directly interact with the bot, just like they do with their friends.

Context Enabled Chatbots - Contextual chatbots are the most advanced kind of conversational bots. They utilize Machine Learning and Artificial Intelligence to remember conversations that happened in the past, with specific users, to learn and grow over time. These chatbots learn with their experiences with the user. Siri, Alexa, Google Assistant are some of the examples of contextual chatbots.

Voice Enabled Chatbots - Voice enabled chatbots like Alexa, Siri creates personalized experience for the users. These chatbots accept user inputs through voice, act upon user request, answer their queries, and perform a number of creative tasks. Businesses can create their own voice-activated chatbot by using text-to-speech (TTS) and voice recognition APIs.

\section{General Chatbot Architecture}

The process starts with a user's request, for example, "What is the meaning of environment?", to the chatbot using a messenger app like Facebook, Slack, WhatsApp,

WeChat or Skype, or an app using text or speech input like Amazon Echo.

After the chatbot receives the user request, the Language Understanding Component parses it to infer the user's intention and the associated information (intent: "translate," entities: [word: "environment"]) Once a chatbot reaches the best interpretation it can, it must determine how to proceed. It can act upon the new information directly, remember whatever it has understood and wait to see what happens next, require more context information or ask for clarification. When the request is understood, action execution and information retrieval take place. The chatbot performs the requested actions or retrieves the data of interest from its data sources, which may be a database, known as the Knowledge Base of the chatbot, or external resources that are accessed through an API call.

\section{Hosted file}

image1.emf available at https://authorea.com/users/360481/articles/482164-chatbots-andvirtual-assistants

Upon retrieval, the Response Generation Component uses Natural Language Generation

(NLG) to prepare a natural language human-like response to the user based on the intent and context information returned from the user message analysis component. A Dialogue Management Component keeps and updates the context of a conversation which is the current intent, identified entities, or missing entities required to fulfill user requests. Moreover, it requests missing information, processes clarifications by users, and asks follow-up questions. For example, the chatbot may respond: "Would you like to tell me as well an example sentence with the word environment?"

\section{Conclusion}

Minimal human interference in the use of devices is the goal of our world of technology.

Chatbots can reach out to a broad audience on messaging apps and be more effective than humans are. At the same time, they may develop into a capable information-gathering. They provide significant savings 
in the operation of customer service departments. With further development of AI and machine learning, somebody may not be capable of understanding whether he talks to a chatbot or a real-life agent.

I consider that this research provides useful Information about the basics of Chatbots. Users can have a better understanding of the concepts.

\section{References}

1. Wikipedia-https://en.wikipedia.org/wiki/Artificial_intelligence

2. acquire.io-https://acquire.io/

3 . SearchEnterpriseAI-https://searchenterpriseai.techtarget.com/definition/natural-languageunderstanding

4. insights.daffodil.com-https://insights.daffodilsw.com/

5. GeeksforGeeks-https://www.geeksforgeeks.org/chatbot-theory-explained/ 